Universidad de Lima

Facultad de Psicología

Carrera de Psicología

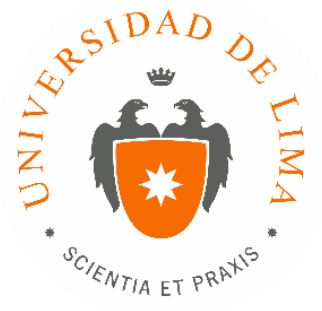

\title{
EXPERIENCIA DE EJECUCIÓN DE UN PLAN DE CAPACITACIÓN ANUAL E IMPLEMENTACIÓN DE UN ERP
}

Trabajo de suficiencia profesional para optar el título profesional de Licenciado en Psicología

\author{
Cynthia Alicia Chuyes Ruiz
}

Código 20111716

$$
\text { Lima - Perú }
$$

Febrero de 2019 


\section{EXPERIENCIA DE EJECUCIÓN DE UN PLAN DE CAPACITACIÓN ANUAL E IMPLEMENTACIÓN DE UN ERP}




\section{TABLA DE CONTENIDO}

INTRODUCCIÓN

CAPÍTULO I: IDENTIFICACIÓN DEL PROBLEMA.................................................

CAPÍTULO II: DESCRIPCIÓN DE LAS ACTIVIDADES REALIZADAS..................9

2.1 Levantamiento de necesidades y armado de planes.........................................9

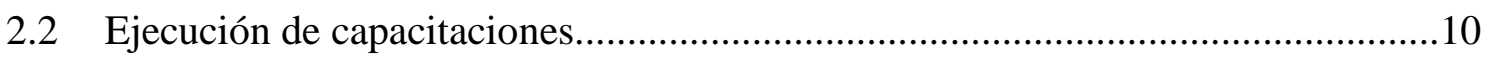

2.2.1 Estructura de contenidos...............................................................10

2.2.2 Ejecución de las capacitaciones (instalación/proyectos)............................11

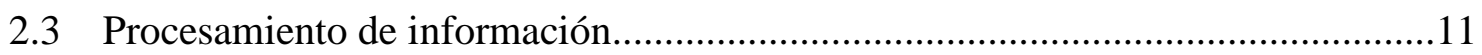

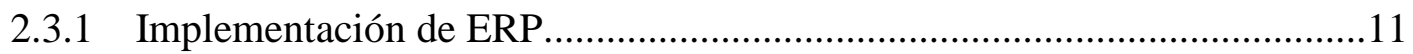

CAPÍTULO III: RESULTADOS DE LA INTERVENCIÓN........................................14

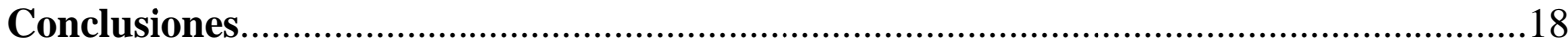

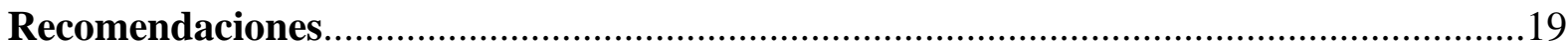

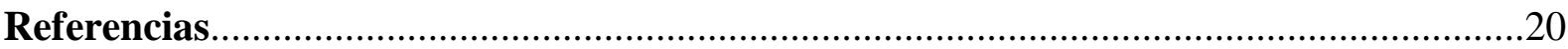




\section{INTRODUCCIÓN}

En los últimos años, se empieza a tomar conciencia sobre la importancia de la capacitación del personal en las nuevas tendencias de recursos humanos; ya que, repercute en la productividad y rentabilidad del negocio como en otras variables trascendentales del mundo organizacional.

Según Chiavenato (2009), la constante capacitación de las personas es el cimiento principal para los programas de mejora. Se trata de uno de los procesos más relevantes de la gestión humana y no desarrollarlo trae consigo conflictos en las relaciones de trabajo, desmotivación en los colaboradores, poca capacidad de competencia, etc.

La finalidad del presente trabajo es recalcar, nuevamente, la importancia de gestionar la capacitación a través de la narración de una experiencia con los planes de capacitación anual en los distintos proyectos e instalaciones de una empresa de construcción peruana. Así mismo, se detalla la implementación de un ERP en parte del proceso de la ejecución del plan. Primero se hará referencia, con teoría relacionada, a los beneficios por los cuales las empresas optan por implementar y ejecutar un plan de capacitación. Se describe los elementos del contexto en el que se manejaba el plan en la empresa, así como los participantes de la experiencia. Seguido de las actividades que se realizaron para el armado y la ejecución del programa. De igual forma, se mencionan las modalidades en que se evaluaron los resultados, que comprenden: evaluaciones escritas al momento y después de las capacitaciones y algunos indicadores. Finalmente, se expondrán conclusiones y recomendaciones desprendidas de lo anterior. 


\section{CAPÍTULO I: IDENTIFICACIÓN DEL PROBLEMA}

El éxito de una organización se basa en la calidad de su gente. Se podrían realizar muy buenos planes, excelentes organigramas y utilizar sofisticados controles contables, y sin embargo, fracasar al tener al personal incorrecto o al no lograr motivar a los equipos. Muchos líderes han tenido éxito incluso con planes, organizaciones o controles deficientes. Triunfaron porque contrataron a las personas indicadas para los puestos correctos y porque supieron motivar, evaluar y desarrollar a dichas equipos (Dessler, 2009). La gestión humana permite tomar acciones encaminadas a facilitar que los recursos humanos contribuyan al logro del negocio, es decir, al logro de la estrategia y metas organizacionales.

Las empresas están en constante evolución y con los avances tecnológicos de los últimos años, se ha activado una avalancha de cambios, ya que se tiene mayor acceso a plataformas que permiten automatizar las tareas. Se abre, entonces, una era de competencia diferente con clientes externos e internos más exigentes. En este contexto, se encuentran brechas cada vez más grandes entre los colaboradores y las demandas reales de Recursos Humanos, aquí es donde la capacitación se vuelve indispensable.

Más compañías optan por gestionar el desarrollo de sus equipos porque es un beneficio valorado por los colaboradores, donde la empresa demuestra la importancia que le da a su recurso humano, aumentando el compromiso y la retención del mismo. Así que, aunque la capacitación aparentemente tenga como principal objetivo eliminar brechas, esta ya no es su única función. El entrenamiento casi siempre ha sido entendido como el proceso mediante el cual se prepara a la persona para que desempeñe con excelencia las tareas específicas del puesto que ocupa. Actualmente la capacitación es un medio que desarrolla las competencias de las personas para que puedan ser más productivas, creativas e innovadoras (Chiavenato, 2009). 
Parra y Rodriguez (2016), afirman que la capacitación surge como consecuencia de la urgencia de preparar a los trabajadores y adaptarse lo más rápido posible pero sin perder de vista los objetivos, la misión y la visión de la organización. Es decir, no se debe dejar de lado los elementos de la cultura del negocio, ya que la capacitación será una herramienta para divulgarla y fortalecerla.

Pero, ¿cómo se logra gestionar la formación con éxito?, según Cardozo (2012), se deben articular un conjunto de recursos y medios de manera estratégica, donde los profesionales de RRHH deben poder dar servicios de formación equivalente a un consultor externo pero con el conocimiento preciso del negocio. El nuevo RRHH no se percibe como costo o gasto sino como activo, habiendo superado a la clásica administración y dirección de personal (Cuesta, 2010).

En el presente trabajo, se expondrá la experiencia de ejecución del plan anual de capacitación interna y la implementación de un ERP para manejar digitalmente el proceso, de acuerdo a los nuevos retos que se iban presentando con el tiempo. Todo lo anterior, sucede en una empresa peruana del rubro de construcción fundada hace 52 años. En el momento de la experiencia, la compañía contaba con 4000 colaboradores aproximadamente, divididos en 10 gerencias y tenía alrededor de 21 proyectos en curso.

Recursos Humanos manejaba un proceso de capacitación interna con un plan anual de cursos (impartidos por los mismos colaboradores especialistas en los temas) para cada proyecto e instalación. Para la empresa, el objetivo de este proceso es tener trabajadores preparados para atender las demandas actuales y de crecimiento, así mismo, permitiéndoles autodesarrollo, acceso a conocimientos y oportunidades que incentiven su desarrollo profesional.

Al ser una empresa dedicada a la ingeniería y construcción, contaba con distintos proyectos y estos, se dividían en 3 tipos o líneas de negocio: obras de infraestructura, edificación y mantenimiento vial; es importante mencionarlo, ya que cada línea de negocio tenía una 
necesidad particular y los proyectos dentro de cada línea de negocio, se encontraban en etapas diferentes; así que eran variables a evaluar para armar los planes.

De todo esto, se encargaba el área de Desarrollo Humano (DH), que estaba conformada por la Jefa de $\mathrm{DH}$, una asistente, un practicante profesional y un practicante pre-profesional. Las capacitaciones dentro del plan se encontraban divididas por módulos o temas: Seguridad, Salud y Medio ambiente, Calidad, RRHH, Logística, Producción y Equipos. Es relevante recalcar que existía un Comité de Capacitación conformado por los líderes de las áreas anteriormente mencionadas (un especialista de cada una); de igual manera, cada línea de negocio contaba con un gerente y cada proyecto, con un ingeniero residente.

Durante el proceso de ejecución del plan, que se verá con más detalle en el siguiente capítulo, la empresa pasó por auditorias externas por parte de SGS para recertificar las ISOS con las que contaba. Como resultado de la auditoría, se observó desorganización en la información, al no encontrarse las evidencias de entrenamiento para demostrar las 4 horas de capacitación obligatorias por colaborador en el rubro, lo que generó una solicitud de acción correctiva (SAC). 


\section{CAPÍTULO II: DESCRIPCIÓN DE LAS ACTIVIDADES Y TAREAS REALIZADAS}

\subsection{Levantamiento de necesidades y armado de planes}

A principio de año, se establecieron las metas organizacionales y la estrategia de negocio. El equipo de DH y el comité de capacitación se reunió con cada gerente de línea de negocio individualmente para evaluar cuáles eran las brechas que su equipo presentaba o podrían presentar para lograr las metas y así determinar las necesidades de capacitación para el año. En algunos casos, los líderes sabían exactamente que capacitaciones querían para sus equipos; en otros, se evaluaba el caso junto con los especialistas del tema (comité de capacitación) y con el equipo de DH para determinar que entrenamiento sería oportuno.

Una vez mapeadas las capacitaciones de acuerdo al tipo y la etapa en la que se encontraba el proyecto, junto con el líder, más el asesoramiento de los especialistas; se procedía a determinar quién impartiría el entrenamiento (ej. Jefe de control de gestión), el grupo poblacional a quien iría dirigido (ej. Operadores, línea de mando) y el mes tentativo a dictarse.

Una vez determinados los datos anteriores por línea de negocio, DH realizó reuniones más especificas, junto con cada ingeniero residente y el encargado de RRHH de cada obra; es decir, una reunión por proyecto para cerrar cada uno de los planes. De esta manera, podían realizar aportes o modificaciones, con conocimiento de la realidad de su proyecto.

Ya que se tenía el plan completo, el equipo de DH ejecutaba la carga de cada plan al sistema intranet donde los encargados de RRHH de los proyectos podían visualizar los detalles de las capacitaciones, puesto que ellos eran los responsables 
de que estas se realizaran. Por su lado, el equipo de DH era el encargado de que el plan de la oficina principal se cumpliera.

Ninguno de los 22 planes eran cargados al sistema intranet, sin antes ser aprobado por el comité de capacitación, el ingeniero residente del proyecto, el gerente de la línea de negocio y finalmente, el gerente de Recursos Humanos. De todas estas validaciones se encargaba el equipo de DH y tardó alrededor de 3 meses (desde diagnóstico hasta aprobación).

En caso surgían necesidades de capacitación imprevistas, eran evaluadas e incluidas en el plan que se actualizaba y se cargaba a intranet nuevamente.

Cada tres meses, se realizaban los comités de capacitación, tanto en la oficina principal como en los distintos proyectos. Consistía en una reunión con los integrantes del comité mencionado, que tenía por finalidad revisar el cumplimiento del plan de capacitación y tomar medidas de acción en caso se presenten desviaciones. En caso no se pudiera cumplir con alguna capacitación en el mes programado, se podía reprogramar en los siguientes 3 meses, siempre dejando constancia y justificación en el "Acta de Reunión de Comité”.

\subsection{Ejecución de capacitaciones}

\subsubsection{Estructura de los contenidos}

Para realizar el contenido de las capacitaciones se tomó en cuenta los diferentes niveles de responsabilidad, habilidades de lenguaje, alfabetismo y riesgos, que permitan el entendimiento adecuado. Cada gerencia o área responsable de brindar la capacitación eran los encargados de armar los contenidos y estructura de los cursos, para luego ser enviados al equipo de DH, quien revisaba, aprobaba y estandarizaba el contenido para ser 
difundido a los instructores tanto en la oficina principal como en proyectos. Cabe resaltar, que estos facilitadores, fueron parte de un "Programa de Instructores" donde desarrollaron las competencias necesarias para cumplir su rol eficientemente.

\subsubsection{Ejecución de las capacitaciones (instalación/proyectos)}

En cuanto a la ejecución de capacitaciones en la instalación de oficina principal, el equipo de $\mathrm{DH}$ se encargó de contactar cada mes a los instructores de las capacitaciones para programar una fecha factible de realizarlas dentro del mes programado.

Una vez coordinada la fecha, DH se comunicó con el área de comunicación para realizar una invitación con un mensaje atractivo, que se envía unos días antes del evento a todas las personas para quienes va dirigida la capacitación. Así mismo, el día del evento, DH gestiona los aspectos logísticos como sala, proyector, coffee break, posición de los invitados, tarjetas de identificaciones, laptop, material de trabajo; todo esto adecuado especialmente a la capacitación y al grupo ocupacional.

En cuanto a las capacitaciones de los 21 proyectos, DH se encargaba únicamente de solucionar dudas que los gestores de RRHH en proyecto tuvieran sobre temas de alcance, instructor, materiales; de todo lo demás se responsabilizaban los gestores de recursos en proyecto.

\subsection{Procesamiento de información}

\subsubsection{Implementación de ERP}

Al finalizar las capacitaciones, se tenía una lista de asistencia donde cada participante firmaba como evidencia de la realización del evento. En la 
oficina principal, el equipo de $\mathrm{DH}$, almacenaba las evidencias en pioners. Por otro lado, los encargados de RRHH en proyectos, tenían la responsabilidad de enviar las listas de asistencia escaneadas por correo electrónico al equipo de DH en la oficina principal, ellos a su vez las organizaban en carpetas. Evidentemente, 21 proyectos con aproximadamente 40 capacitaciones cada uno, generaba mucha documentación escaneada y la dificultad de ubicar cualquier dato que se necesitara en particular. Cuando los auditores (tanto internos como externos) solicitaban las capacitaciones de algún colaborador en específico, era casi imposible obtener la evidencia inmediatamente; ya que había que buscar en la carpeta del proyecto o en el peor de los casos, por la premura de las actividades, no se guardaban los escaneos en las carpetas y se tenía que buscar en los correos.

Es así como surgió la necesidad de utilizar un sistema que consolide todos los documentos de manera organizada y que permita el acceso a los responsables de RRHH desde proyectos, para que cada uno pueda cargar sus evidencias y descentralizar esa función de la oficina principal. El equipo de DH coordinó una reunión con el área de Sistemas para evaluar la posibilidad de armar módulos en SAP que permitan realizar lo mencionado anteriormente.

En SAP, se crearon las carpetas "interno" haciendo referencia a capacitación interna por cada proyecto. Dentro de esta carpeta se generaron módulos por cada tema de capacitación (como están divididas en el plan) y dentro de los temas, se crearon las carpetas con el nombre de cada capacitación. Es decir, los módulos estaban generados de acuerdo al plan 
anual, ya preparados solo para cargar la fecha en que se dio la capacitación, los asistentes y adjuntar las evidencias.

Una vez que el área de Sistemas procede a la implementación de los módulos, se pasa a un periodo de corridas y pruebas. Cuando se han realizado las modificaciones necesarias, se cambia el procedimiento formalmente con el área del Sistema Integrado de Gestión y se da paso al lanzamiento de la nueva metodología y al entrenamiento de los responsables de RRHH en proyectos, mediante videollamadas con pantallas compartidas.

Entonces, después del cumplimiento de las capacitaciones, los encargados de $\mathrm{RRHH}$ en proyectos registraban a los colaboradores en el módulo de SAP y anexaban las evidencias. Después, el equipo de DH, validaba y daba seguimiento a las cargas de los proyectos para realizar el indicador de capacitación

Una vez que todas las evidencias se encontraban en el sistema, el equipo de DH se encargaba mensualmente de procesar las datas resultantes de SAP para obtener indicadores. 


\section{CAPÍTULO III: RESULTADOS DE LA INTERVENCIÓN}

En cuanto a los resultados de las capacitaciones, se pueden realizar de distintas maneras.

Kirkpatrick (citado en Cano, 2015) diseñó un modelo de evaluación de acciones formativas que se aplica en distintos ámbitos, no solo en el sector educativo sino también en el organizacional. Este es un modelo niveles en los que se evalúa a quien recibe el entrenamiento o capacitación. Primero, se evalúa la satisfacción del curso inmediatamente después de realizarse. En el segundo nivel, se mide el aprendizaje inmediato con un control al finalizar el curso. El tercer y cuarto nivel de comportamiento se enfoca en la práctica de lo aprendido y se debe de medir transcurridos de 3 a 5 meses de concluido el curso, ya que solo a partir de ese tiempo se puede confirmar que el conocimiento ha sido incorporado.

Similar al modelo anterior, en el caso de esta empresa, primero se realizaba una evaluación de la satisfacción de la capacitación después de realizado el evento; consistía en una evaluación escrita sobre el contenido del curso, el desempeño del capacitador, el material utilizado, etc.

Como segundo nivel, se medía la retención de los conocimientos impartidos, consistía en una evaluación escrita u oral basada en una rúbrica de evaluación. Este examen se aplicaba a una cantidad específica de participantes, para la cual se utilizaba la fórmula del cálculo del tamaño muestral para poblaciones finitas. Estas calificaciones eran registradas por el instructor en el formato de asistencia y se entregaban junto con los exámenes al equipo de DH (oficina principal) o a los encargados de RRHH (proyectos), quienes cargaban toda esta información a los módulos de SAP junto con los anexos ya mencionados. Todos los colaboradores obtuvieron una calificación mayor a 14 en las capacitaciones del año en todos los proyectos. 
Así mismo, como último nivel, se realizaba la evaluación de la eficacia de la capacitación, que debía evaluarse dentro de los 6 meses posteriores a su realización. Esta evaluación servía para poder verificar la aplicación del entrenamiento en el puesto de trabajo según lo indicado en el plan anual de capacitación. Cabe resaltar que no todas las capacitaciones aplicaban a la medición de eficacia. El comité central de capacitación definía el impacto del entrenamiento calificando del 1 al 4 (siendo 1 menor impacto) en: la empresa, en el proyecto, en SSMA (seguridad, salud y medio ambiente), en productividad y en gestión y soporte. Se obtenía un promedio de las anteriores calificaciones y si es que el total era más de 3, se debía medir la eficacia. Todo esto estaba indicado en el plan de capacitación de cada proyecto o instalación. Se podía evaluar mediante 3 métodos: pruebas de demostración de habilidades, observación directa o exámenes escritos. El facilitador de la capacitación indicaba cuál era el método oportuno y la cantidad de personas a evaluar era un porcentaje de los asistentes indicado por el equipo de DH que se obtenía de la siguiente manera: si participaron de 0 a 20 personas, se medía eficacia a 50\%; si participaron de 21 a 50 personas, 25\%; si participaron de 51 a 100 personas, $15 \%$ y si participaron a partir de 101 personas, $10-15 \%$.

Además de estas evaluaciones, se pudo medir los resultados de acuerdo al indicador de participación y de cumplimiento. Al finalizar el mes, cada proyecto debía haber registrado y cargado las evidencias de las capacitaciones realizadas en SAP, de esta manera el equipo de DH podía correr una data donde salían todos los participantes, de todas las capacitaciones, de todos los proyectos. Una vez procesada y trasformada a tablas dinámicas, se podía visualizar el indicador mensual de participación y de cumplimiento de acuerdo al corte de preferencia: por proyecto, por línea de negocio, por puestos, etc.

De manera trimestral, estos datos eran cargados por la Jefa de DH a una web, donde los visualizaba el Gerente General. También, se realizaban reuniones, con los gerentes de líneas 
de negocios para verificar como iban sus proyectos en cuanto a capacitación, de esta manera iban revisando las causas de posibles incumplimientos y acciones de mejora.

Se obtuvo un promedio mayor del $80 \%$ de participación en todo el plan anual por proyecto. Cuando se obtuvo menos del $80 \%$ de participación en la capacitación, se reprogramó en los siguientes 3 meses hasta alcanzar el porcentaje. Así mismo, se obtuvo un $100 \%$ de cumplimiento de los planes de capacitaciones por proyecto; en caso no se haya realizado alguna capacitación por alguna eventualidad o coyuntura del proyecto, esta se tenía que especificar con la debida justificación en el acta trimestal de capacitación de su proyecto colgado en SAP.

Sobre los resultados de la implementación del ERP al procedimiento de capacitación, se pudo levantar la solicitud de acción correctiva (SAC) ya que, se sabía exactamente en que lugar estaban las evidencias de los colaboradores por proyecto y disminuyó el tiempo destinado a encontrar las evidencias (listas de asistencia y exámenes) que solicitaban en auditorias internas y externas. Se redujo el tiempo en que se procesaba la información para obtener el indicador de capacitación y estos eran más reales, puesto que el sistema no permitía duplicidad de nombres, hacía cruce con la planilla del proyecto, etc

En cuanto a la aceptación o resistencia del cumplimiento del plan, es importante mencionar que al ser una empresa de construcción, se percibe resistencia sobre la aplicabilidad de temas blandos como es capacitación. Al principio costó que los gerentes de líneas de negocios e ingenieros residentes destinaran un tiempo para las reuniones de diagnóstico de necesidades, para las revisiones de avances, etc y se notaba cierta molestia cuando se mencionaba que los gestores de RRHH e instructores de sus proyectos debían dar más importancia al cumplimiento del plan y no solo a planillas, marcación u otras funciones importantes de sus áreas. Así mismo, en alguna oportunidad, se vio la necesidad de que el Gerente de RRHH llamara a gestores de RRHH en proyectos para cuestionar porque no se estaban haciendo las 
cargas a SAP a tiempo. Finalmente, se logró que los porcentajes y calificaciones fueron los deseados.

De igual manera, con la implementación de SAP, inicialmente los encargados de RRHH preferían continuar enviando las evidencias de capacitación por correo. Se mostraban renuentes a cualquier cambio que implicara el ERP porque tomaba tiempo aprender las transacciones y las secuencias; llamaban con frecuencia al equipo de DH para que los acompañara en el proceso por teléfono. Se realizaron manuales sencillos en word con capturas de pantalla de SAP, paso a paso, para cada acción que tuvieran que realizar como: crear eventos, inscribir participantes, anexar documentos, etc. Con lo cual se redujo el tiempo que DH destinaba a explicar cada mes durante el periodo de aprendizaje.

El mundo organizacional cambia cada vez más rápido y la disciplina psicológica se ve involucrada en la necesidad de cubrir las brechas que generen estos avances acelerados; así como también, se ve en la obligación de incorporar estos desarrollos a sus propios procesos, permitiendo hacerlos más ágiles y precisos. 


\section{CONCLUSIONES}

- El desarrollo de este plan permitió comprobar que si se realiza un plan de capacitación alineado a la misión, visión y metas organizacionales, trae resultados beneficiosos y es posible adaptarlo a la realidad del negocio. Como se ha podido observar, no hubiera funcionado un único plan como en otras empresas, ya que cada proyecto tenía necesidades distintas y se encontraba en etapas de ejecución diferentes.

- De igual manera, es necesario destinar un tiempo a determinar la forma en que se estructurarán los contenidos para ser impartidos; ya que, esto va a impactar en buena medida en el éxito del plan y en la incorporación de los conocimientos.

- El hecho de tratarse de un rubro "hard", duplica la atención y el detalle que se le da a cómo trasmitir, enseñar, supervisar, evaluar etc; puesto que quizás no es tan evidente la trascendencia de los procesos de RRHH para los líderes de proyectos en una empresa de construcción e ingeniería.

- Esta experiencia, también, permite recordar y resaltar, que toda implementación trae consigo un periodo de adaptación y es posible que en ese tiempo se deban hacer mejoras acorde a la realidad del contexto; lo cual no debe desalentar la implementación, por el contrario, esto permitirá tener una herramienta o procedimiento mucho más completo y preciso. 


\section{RECOMENDACIONES}

A continuación se detallaran las recomendaciones:

- Realizar todo el procedimiento del plan de capacitación nuevamente, actualizándolo con las metas de negocio del año y por supuesto, a la misión, visión y valores.

- Llevar a cabo presentaciones trimestrales de las encuestas de satisfacción de cada capacitación para poder dar retroalimentación a los facilitadores sobre los contenidos y las metodologías de enseñanza. Así mismo, estos resultados podrían ser útiles en el "programa de instructores".

- Realizar actividades que fomenten la cultura empresarial donde se resalte la importancia que la empresa le da a la capacitación, al desarrollo de sus colaboradores y los beneficios de esto. Para tener líderes más involucrados que bajen las ideas a sus equipos y así, generar un cambio de percepción sobre las tareas necesarias para cumplir el plan de capacitación, que sean vistas como un beneficio y no como trabajo innecesario.

- Llevar a cabo una reunión a fin de año con los líderes de proyectos, el comité de capacitación y el equipo de DH en orden de recoger sus percepciones y recomendaciones para el planeamiento del programa del siguiente año. 


\section{REFERENCIAS}

Cano, E. (2015). Evaluación de la formación. Algunas lecciones aprendidas y algunos retos de futuro. Educar, 51(1), 109-125. doi: http://dx.doi.org/10.5565/rev/educar.684

Cardozo, P. (2012). Desarrollo humano de las organizaciones. Buenos Aires: Temas Grupo Editorial.

Chiavenato, I. (2009). Gestión del talento humano (3ra ed.). Colombia: McGraw Hill.

Cuesta, A. (2010). Gestión del talento humano y del conocimiento. Bogotá: ECOE Ediciones

Dessler, G. (2009). Administración de recursos humanos (11a ed.). Recuperado de: https://www.academia.edu/13314554/Administracion_de_recursos_humanos_11va_ edicion_-_Gary_Dessler.

Parra, C. y Rodríguez, F. (2016). La capacitación y su efecto en la calidad dentro de las organizaciones. Revista de Investigación, Desarrollo e Innovación, 6(2), 131-146. doi: http://dx. doi.org/10.19053/20278306.4602 